\title{
A NOTE ON ANOSOV DIFFEOMORPHISMS
}

\author{
BY ROBERT J. SACKER ${ }^{1}$ AND GEORGE R. SELL ${ }^{2}$
}

Communicated by Walter Gottschalk, August 28, 1973

1. Introduction. In this note we shall study a class $\Gamma$ of diffeomorphisms on a compact $n$-dimensional manifold $M$. The class $\Gamma$ will include all diffeomorphisms $F$ with the property that the periodic points of $F$ are dense in $M$. Our main theorem will give a characterization of those diffeomorphisms in $\Gamma$ that are Anosov diffeomorphisms.

2. Statement of results. Let $F: M \rightarrow M$ be a diffeomorphism on a compact $n$-dimensional manifold $M$ and let $D F: T M \rightarrow T M$ be the induced derivative mapping on the tangent bundle of $M$. The mapping $F$ is said to be an Anosov diffeomorphism if the tangent bundle can be decomposed into a continuous Whitney sum $T M=E^{s}+E^{u}$, such that

(i) $E^{s}$ and $E^{u}$ are invariant under $D F$;

(ii) $D F: E^{s} \rightarrow E^{s}$ is contracting, i.e., there exist positive constants $K$ and $\lambda, \lambda<1$, such that

$$
\left\|D F^{m}(v)\right\| \leqq K \lambda^{m}\|v\|
$$

for all $v \in E^{s}$ and $m \in Z^{+}$;

(iii) $D F: E^{u} \rightarrow E^{u}$ is expanding, i.e., there exist positive constants $k$ and $\mu, \mu>1$, such that

$$
\left\|D F^{m}(v)\right\| \geqq k \mu^{m}\|v\|
$$

for all $v \in E^{u}$ and $m \in Z^{+}$, cf. [1], [3], and [6].

Since $D F$ is a homeomorphism, the composed mapping $D F^{m}$ is defined for all $m \in Z$, and this defines a discrete flow on $T M$. Similarly $F^{m}$ is a discrete flow on $M$, and these flows commute with the natural projection $p: T M \rightarrow M$. Now let $\Gamma$ denote the collection of all diffeomorphisms $F: M \rightarrow M$ such that the union of the minimal sets of the flow $F^{m}$ is dense in $M$. For example, if the periodic points of $F$ are dense in $M$, then $F \in \Gamma$.

For any diffeomorphism $F: M \rightarrow M$ we define the sets $\mathscr{B}, \mathscr{S}, \mathscr{U}$ in

AMS (MOS) subject classifications (1970). Primary 58F15; Secondary 34C35.

${ }^{1}$ The first author was partially supported by U.S. Army Grant DA-ARO-D-31-12471-G176.

${ }^{2}$ The second author was partially supported by NSF Grant GP-38955. 
the tangent bundle $T M$ by

$$
\begin{aligned}
& \mathscr{B}=\left\{v \in T M:\left\|D F^{m}(v)\right\| \text { is bounded uniformly for } m \in Z\right\}, \\
& \mathscr{S}=\left\{v \in T M:\left\|D F^{m}(v)\right\| \rightarrow 0 \text { as } m \rightarrow+\infty\right\}, \\
& \mathscr{U}=\left\{v \in T M:\left\|D F^{m}(v)\right\| \rightarrow 0 \text { as } m \rightarrow-\infty\right\} .
\end{aligned}
$$

In the theorem, which we next state, the sets $\mathscr{S}$ and $\mathscr{U}$ will take the role of $E^{s}$ and $E^{u}$. However we want to emphasize that the contracting and expanding properties described by (1) and (2) are not included in the definition of $\mathscr{S}$ and $\mathscr{U}$; they follow as one of the consequences of our theorem.

Theorem. Let $F: M \rightarrow M$ be a diffeomorphism on a compact $n$-dimensional manifold $M$ and assume that $F \in \Gamma$. Then $F$ is an Anosov diffeomorphism if and only if $\mathscr{B}=T M_{0}$, the zero section of TM. Moreover, in this case $\mathscr{S}$ and $\mathscr{U}$ are subbundles of $T M$ which are invariant under the flow $D F^{m}, T M=\mathscr{S}+\mathscr{U}$ (Whitney sum), DF: $\mathscr{S} \rightarrow \mathscr{S}$ is contracting, and $D F: \mathscr{U} \rightarrow \mathscr{U}$ is expanding.

3. Outline of proofs. The proof of this theorem is included in a paper in which we study the general problem of (discrete and continuous) linear flows on vector bundles [4]. The results in this paper also have important applications in the theory of linear differential equations with almost periodic coefficients.

The proof of the necessity of the condition $\mathscr{B}=T M_{0}$ follows directly from the definition of an Anosov diffeomorphism. The proof of the sufficiency of this condition is accomplished as follows: For $y \in M$ define the fibers

and define

$$
\mathscr{S}(y)=p^{-1}(y) \cap \mathscr{S}, \quad \mathscr{U}(y)=p^{-1}(y) \cap \mathscr{U},
$$

$$
\begin{aligned}
& A^{+}=\left\{v \in \mathscr{S}:\left\|D F^{m}(v)\right\| \leqq 1 \text { for all } m \in Z^{+}\right\}, \\
& A^{-}=\left\{v \in \mathscr{U}:\left\|D F^{-m}(v)\right\| \leqq 1 \text { for all } m \in Z^{+}\right\} .
\end{aligned}
$$

Note that $\mathscr{S}(y)$ and $\mathscr{U}(y)$ are linear subspaces of $T_{y} M$, the tangent space of $M$ at $y$.

Step 1. $A^{+}$and $A^{-}$are compact sets in $T M$ and there is a $\sigma, 0<\sigma<1$, such that $\{v \in \mathscr{S}:\|v\| \leqq \sigma\} \subseteq A^{+}$, and $\{v \in \mathscr{U}:\|v\| \leqq \sigma\} \subseteq A^{-}$.

Step 2. $\mathscr{S}$ and $\mathscr{U}$ are closed sets and $\left\|D F^{m}(v)\right\| \leqq \sigma^{-1}\|v\|$ for all $v \in \mathscr{S}$ and $m \in Z^{+}$, and $\left\|D F^{-m}(v)\right\| \leqq \sigma^{-1}\|v\|$ for all $v \in \mathscr{U}$ and $m \in Z^{+}$.

Step 3. The functions $\operatorname{dim} \mathscr{S}(y)$ and $\operatorname{dim} \mathscr{U}(y)$ are upper semicontinuous functions of $y$.

Step 4. $\mathscr{S}$ and $\mathscr{U}$ are invariant under $D F$ and $D F: \mathscr{S} \rightarrow \mathscr{S}$ is contracting and $D F: \mathscr{U} \rightarrow \mathscr{U}$ is expanding. 
Step 5. If $y$ belongs to a minimal set for the flow $F^{m}$ on $M$, then

$$
\mathscr{S}(y)+\mathscr{U}(y)=T_{y} M .
$$

The proof of Step 5 uses essentially a duality argument and reduces to examining properties of the intersection number of singular chains in $R^{n}$.

Since (3) holds over a dense set in $M$ we show, using Steps 2 and 3, that $\mathscr{S}$ and $\mathscr{U}$ are subbundles and that $T M=\mathscr{S}+\mathscr{U}$ (Whitney sum).

REMARKS. (1) By using a known property of Anosov diffeomorphisms [7], the condition $\mathscr{B}=T M_{0}$, for $F \in \Gamma$, then implies that the periodic points of $F$ form a dense set in $M$. Also see [3, p. 116].

(2) If one does not assume that the set $N=$ \{union of the minimal sets of the flow $\left.F^{m}\right\}$ is dense in the manifold $M$, then it still follows, using our general theory [4], that one gets a corresponding splitting of the tangent bundle over the closure $\bar{N}$.

We show in [5] that this splitting can be extended to all of $M$ provided the dimension of the fiber $\mathscr{S}(y)$ is the same over every minimal set. This fact has also been discovered independently by R. Mañé Ramirez [2] by different techniques.

\section{REFERENCES}

1. D. V. Anosov, Roughness of geodesic flows on compact Riemannian manifolds of negative curvature, Dokl. Akad. Nauk SSSR 145 (1962), 707-709=Soviet Math. Dokl. 3 (1962), 1068-1070. MR 26 \#716.

2. R. Mañé Ramirez, Persistent manifolds are normally hyperbolic (to appear).

3. Z. Nitecki, Differentiable dynamics, M.I.T. Press, Cambridge, Mass., 1971.

4. R. J. Sacker and G. R. Sell, Existence of dichotomies and invariant splittings for linear differential systems. I, J. Differential Equations (to appear).

5. - Existence of dichotomies and invariant splittings for linear differential systems. II (to appear).

6. S. Smale, Differentiable dynamical systems, Bull. Amer. Math. Soc. 73 (1967), 747-817. MR 37 \#3598.

7. P. Walters, Anosov diffeomorphisms are topologically stable, Topology 9 (1970), 71-78. MR 40 \#8069.

Department of Mathematics, University of Southern California, los ANGeles, California 90007

School of Mathematics, University of Minnesota, Minneapolis, Minnesota 55455 